\title{
Comparison of the Incidence of Complications among the Different Techniques of Laparoscopic Approach: Experience in a General Surgery Service in a Private Hospital
}

\author{
Mauricio Valdez-Durón ${ }^{1}{ }^{*}$, Jorge Fernández-Álvarez¹, Alberto Manuel González-Chávez1, \\ Francisco Ángel Vega-Romero ${ }^{2}$, Ketzalcoatl Tecomahua-Román' ${ }^{1}$, Antonio García-Ruiz ${ }^{3}$ \\ ${ }^{1}$ Hospital Español de México, Mexico City, Mexico \\ ${ }^{2}$ Hospital General Xoco, Mexico City, Mexico \\ ${ }^{3}$ Hospital Central Militar, Mexico City, Mexico \\ Email: *drvaldezduron@yahoo.com
}

How to cite this paper: Valdez-Durón, M., Fernández-Álvarez, J., González-Chávez, A.M., Vega-Romero, F.Á., Tecomahua-Román, K. and García-Ruiz, A. (2018) Comparison of the Incidence of Complications among the Different Techniques of Laparoscopic Approach: Experience in a General Surgery Service in a Private Hospital. Surgical Science, 9, 227-232.

https://doi.org/10.4236/ss.2018.97027

Received: May 31, 2018

Accepted: July 14, 2018

Published: July 17, 2018

Copyright $\odot 2018$ by authors and Scientific Research Publishing Inc. This work is licensed under the Creative Commons Attribution International License (CC BY 4.0).

http://creativecommons.org/licenses/by/4.0/ c) (i) Open Access

\begin{abstract}
Introduction: At least four techniques or approaches to minimize the risk of injury have been introduced. The correlation between techniques and the complication rate is controversial. The objective of the study is to report the incidence of laparoscopic entry associated injury, in relation to the used technique. Material and Methods: A retrospective study was conducted at the Hospital Español, in a 12-month period (2013). The medical records of all patients who underwent laparoscopic surgery were reviewed. Demographic and surgical variables were analyzed, comparing the results statistically through an analysis of variance (ANOVA). Results: A total of 792 cases were analyzed. The surgeries performed were: cholecystectomies (42\%), appendectomies (26\%), diagnostic laparoscopies (20\%), ventral or inguinal hernioplasty (9\%) and fundoplications (3\%). The preferred approach was Hasson's (79\%) and Veres's needle technique (14\%), followed by classic closed (14\%) and Palmer technique (2\%). We documented six cases of laparoscopic entry associated injury $(0.76 \%)$, four associated with the Hasson technique, one with Veress's needle technique and one with direct approach technique were reported. The statistical analysis was performed considering the different techniques and their relation with the injuries. Analysis of variance did not reach statistical significance $(p=0.31)$. Conclusions: Among our data, the number of laparoscopic entry associated injuries, was less than $1 \%$, and has a similar frequency to the reported literature. We did not find significant differences between the four laparoscopic entry techniques. There remains no clear evi-
\end{abstract}


dence as to the optimal form of laparoscopic entry in the low-risk patient.

\section{Keywords}

Laparoscopic Surgery, Pneumoperitoneum, Complications

\section{Introduction}

Although laparoscopic approach is currently the choice to perform common abdominal surgeries [1] [2] [3], its use requires creation of a pneumoperitoneum by any of the four already described techniques. That is, 1) classic closed technique with a Veres needle through the umbilicus, 2) Palmer closed technique, with a Veres needle through the left hypochondrium, 3) Hasson or open technique, which involves entering the cavity through a minilaparotomy incision, or 4) Direct technique, inserting a port through the abdominal wall without previous insufflation. Associated injuries during laparoscopic approach are particularly meaningful because of its potential to increase patient's morbidity [4]. Although all the techniques used for the creation of the pneumoperitoneum represent a risk to injure the intra-abdominal organs, it is still discussed in the literature, which of the four is the safest [5].

The aim of the present study is to report the incidence of laparoscopic entry associated injury in each of the four described techniques during a 12 months period, as well as the repercussions for the patient and management in a private academic hospital.

\section{Material and Methods}

We present a retrospective, transversal, observational and descriptive study; at Hospital Español de México, from January 1 to December 31, 2013 (12 months), in which all patients of the general surgery service with laparoscopic entry associated injury history during that period of time, were included. Patients were divided according to the technique employed to create a pneumoperitoneum: 1) Classic closed technique (Veres needle), 2) Palmer closed technique, 3) Hasson or Open technique, and 4) Direct technique. Incomplete clinical histories or those where technique to create pneumoperitoneum was not specified, were excluded. Demographic variables were considered (age, gender, type and urgency of surgery) as well as the different surgical procedures used (surgical technique to create pneumoperitoneum and lesion management when appeared). "IBM-SPSS Statistics" software was used for statistical analysis of the four groups, to determine percentages, central tendency and dispersion measures as well as analysis of variance (ANOVA) of the associated complications for the approach technique.

\section{Results}

During the evaluated period at Hospital Español de México, 871 laparoscopic 
surgeries were performed. Seventy-nine clinical records were excluded, since the technique to create the pneumoperitoneum was not described. Therefore, the present study just included 792 patients (see Figure 1).

Demographic analysis revealed $67 \%$ women and $33 \%$ men, with a mean age of 42 years-old (rate 16 - 94 years) and 39 years-old (rate 16 - 86 years) respectively. $40 \%$ of the surgeries were in patients within the $2^{\text {nd }}$ and $3^{\text {rd }}$ decade of life, $57 \%$ were elective surgeries and $43 \%$ of them were emergency surgeries. According to the type of surgery, $42 \%$ were cholecystectomies, $26 \%$ appendectomies, $20 \%$ diagnostic laparoscopies, $9 \%$ hernioplasties (ventral or inguinal) and 3\% fundoplications. Related to the technique used to create pneumoperitoneum; 113 cases (14\%) used the classic closed technique with Veres needle (Group 1), 14 cases (2\%) used Palmer closed technique (Group 2), 627 cases (79\%) used open Hasson technique (Group 3) and 38 cases (5\%) used direct closed technique (Group $4)$.

From all patients treated $(n=792)$, just six cases reported complications when establishing pneumoperitoneum (incidence $0.76 \%$ ). All of them were transoperatively detected. Among them, four happened with Hasson technique $(\mathrm{n}=627$, $0.63 \%)$, one with closed classic technique $(n=113,0.88 \%)$ and another one with the direct technique $(\mathrm{n}=38,2.63 \%)$. Palmer's closed technique revealed no complications during the pneumoperitoneum creation. Depending on type of complications, four were small bowel perforations handled with primary closure with sutures: three of them laparoscopically and one by exteriorization of the

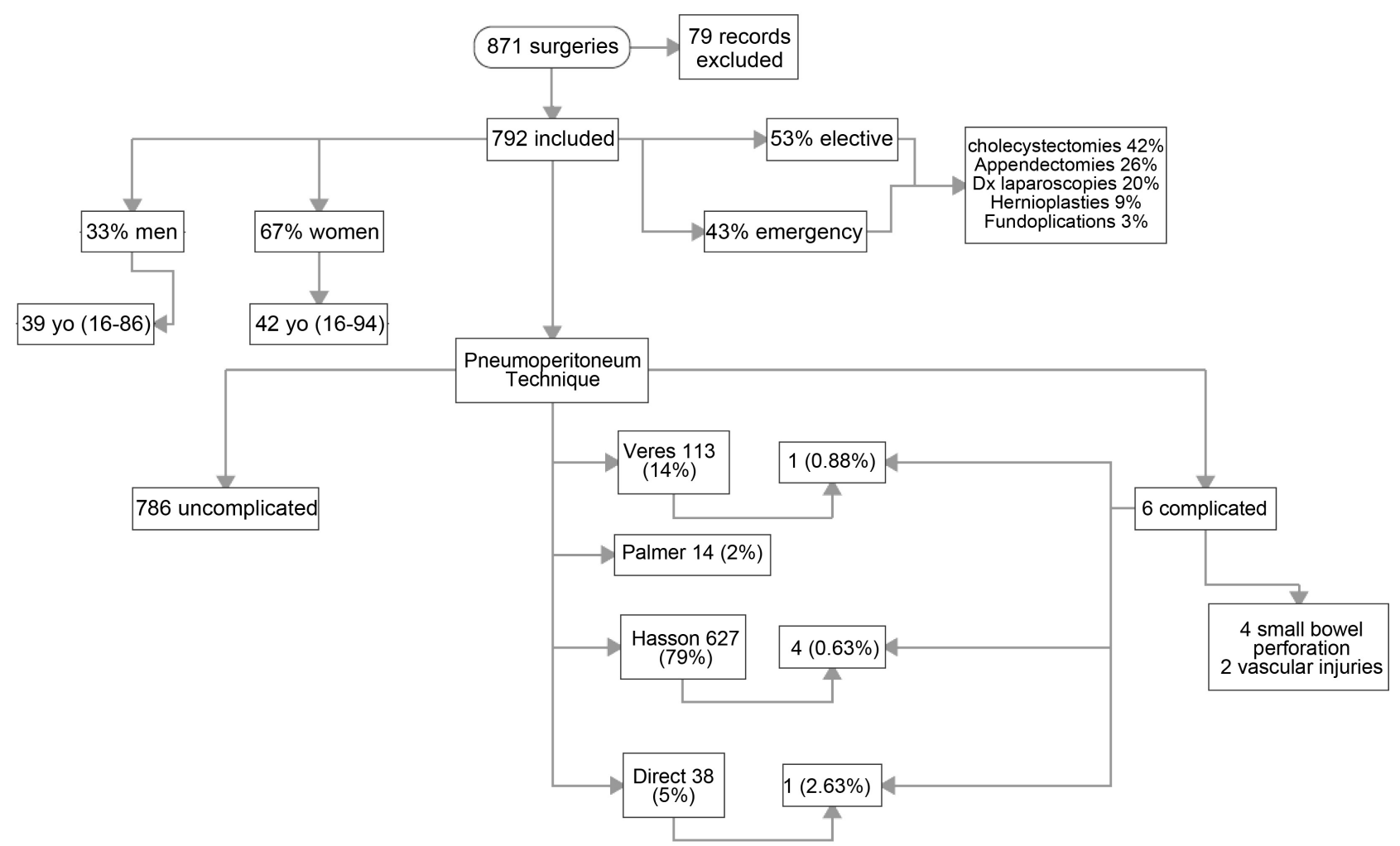

Figure 1. Demographic and surgical variables. Correlation between techniques and the complication rate. 
affected segment through widening the umbilicus incision. Besides, two vascular injuries occur, both cases in the right iliac artery, which required immediate conversion to open surgery and sutured repair of perforation without graft application. No sequela was detected when patients were discharged. Analysis of variance (ANOVA) of complications incidence in the four techniques to create pneumoperitoneum, resulted no statistically significant $(p=0.31)$.

\section{Discussion}

Since 1917, when Hungarian pneumologist Jànos Veres proposed his beveled needle for thoracocentesis, risks of complications for its use were recognized. This puncture technique was later used in abdomen to obtain peritoneal fluid samples and afterwards it was adapted to insufflate the pneumoperitoneum, required for laparoscopies. In order to decrease risks, in 1918 Otto Goetz, from Germany, proposed a change to the technique by means of an automatic needle designed by himself to create the pneumoperitoneum [6]. After that in 1970, an American gynaecologist from Egypt, Harith Hasson, proposed a new technique called "open laparoscopy" by the insertion of a blunt cannula, which allowed insufflation through a $12 \mathrm{~mm}$ incision; done under direct visual control to reduce risks of a blind puncture. In 1974, French gynaecologist Roul Palmer, promoted alternative sites for Veres needle insertion, creating pneumoperitoneum in patients with a previous abdominal surgery, defining the left upper quadrant as the safest site for this purpose [7]. In 1978, American gynaecologist James Ray Dingfelder proposed direct insertion of the first trocar through abdominal wall, without previous insufflation of the pneumoperitoneum, arguing some advantages over previous techniques, specially, that it was easier and faster, causing less subcutaneous emphysema and with similar incidence of complications on its use [8].

As previously mentioned, one of the most important steps in laparoscopic surgery is the access into the abdominal cavity. Several techniques have been described to create pneumoperitoneum; looking for a safest way to achieve it, as this step is one of the most dangerous in laparoscopic approach and could affect the patient, even fatally. However, no technique until now has demonstrated to be better in relation to complication incidence to create pneumoperitoneum for laparoscopic surgery [9] [10].

Complication reports in literature are very similar to the ones presented in our population (0.76\%) [1] [4] [10]. Total incidence of vascular lesions was $0.13 \%$, higher to the reported in general literature, but with $0 \%$ mortality.

Through history, the most important contributions to laparoscopic surgery have been done by gynaecologists who, presumably were the first ones to go on this field and thus, develop most of the instruments used nowadays [6] [7] [8] [9]. For this reason, most papers about laparoscopy have been done by themselves, preferring direct trocar approach arguing less complication incidence compared to the other procedures described [9]. 
Our experience in complication incidence to create pneumoperitoneum was similar within the different approaches. Despite surgeon's trend to use Veres needle (umbilical or Palmer's point) or open approach, in the present study and in the rest of the literature, there is no significant difference supporting that direct trocar approach to abdominal cavity could increase complication risk when creating pneumoperitoneum.

Nevertheless, we should consider carefully this results as in the present trial other important variables were not analyzed like body mass index, presence or number of previous surgeries and abdominal wall hernias; these conditions are important to determine an optimal approach and provide a better patient's safety.

\section{Conclusion}

The best approach for pneumoperitoneum placement should be the one we could manage and the only way to be an expert is continuous practice. Although, current literature shows similar results to the ones obtained in this trial, related to associated lesions for pneumoperitoneum creation, it is not possible to set if there is the same risk for all types of complications and approaches. Larger samples of each type of approach are required in order to show, statistically, which method is safest for pneumoperitoneum placement, thus, providing security to the patient with these kind of procedures.

\section{References}

[1] Bonrath, E.M., Dedy, N.J., Zevin, B. and Grantcharov, T.P. (2014) International Consensus on Safe Techniques and Error Definitions in Laparoscopic Surgery. Surgical Endoscopy, 28, 1535-1544. https://doi.org/10.1007/s00464-013-3348-y

[2] Lal, P., Singh, L., Agarwal, P.N. and Kant, R. (2004) Open Port Placement of the First Laparoscopic Port: A Safe Technique. Journal of the Society of Laparoendoscopic Surgeons, 8, 364-366.

[3] Vilos, G.A., Vilos, A.G., Abu-Rafea, B., Hollett-Caines, J., Nikkhah-Abyaneh, Z. and Edris, F. (2009) Three Simple Steps during Closed Laparoscopic Entry May Minimize Major Injuries. Surgical Endoscopy, 2, 758-764.

https://doi.org/10.1007/s00464-008-0060-4

[4] Altun, H., Banli, O., Karakoyun, R., Boyuk, A., Okuducu, M., Onur, E. and Memisoglu, K. (2010) Direct Trocar Insertion Technique for Initial Access in Morbid Obesity Surgery: Technique and Results. Surgical Laparoscopy Endoscopy \& Percutaneous Techniques, 20, 228-230. https://doi.org/10.1097/SLE.0b013e3181ec6667

[5] Fanning, J., Shah, M. and Fenton, B. (2011) Reduced-Force Closed Trocar Entry Technique: Analysis of Trocar Insertion Force Using a Mechanical Force Gauge. Journal of the Society of Laparoendoscopic Surgeons, 15, 59-61. https://doi.org/10.4293/108680811X13022985131219

[6] Nezhat, C. (2011) Nezhat's History of Endoscopy: A Historical Analysis of Endoscopy's Ascension since Antiquity. Endo Press, Tuttingen, Germany.

[7] Palmer, R. (1974) Safety in Laparoscopy. Journal of Reproductive Medicine, 13, 1-5. 
[8] Dingfelder, J.R. (1978) Direct Laparoscopic Trocar Insertion without Prior Pneumoperitoneum. Journal of Reproductive Medicine, 21, 45-47.

[9] Agresta, F., Mazzarolo, G., and Bedin, N. (2012) Direct Trocar Insertion for Laparoscopy. Journal of the Society of Laparoendoscopic Surgeons, 16, 255-259. https://doi.org/10.4293/108680812X13427982376383

[10] Sasmal, P.K., Tantia, O., Jain, M., Khanna, S. and Sen, B. (2009) Primary Access-Related Complications in Laparoscopic Cholecystectomy via the Closed Technique: Experience of a Single Surgical Team over More than 15 Years. Surgical Endoscopy, 23, 2407-2415. https://doi.org/10.1007/s00464-009-0437-Z 